\title{
A DFT/TD-DFT Study on the Molecular Structure Absorption and Fluorescence Spectra of Gas/Solution Phases Adenosine 5'-triphosphate Molecule
}

\author{
Yavuz EKINCIOGLU ${ }^{1}$, Hamdi Sukur KILIC ${ }^{1,2,3, *}$ (i) Omer DERELI $^{4}$ (D) \\ ${ }^{1}$ Selçuk University, Department of Physics, Faculty of Science, 42031, Konya, Turkey \\ ${ }^{2}$ Selçuk University, Directorate of High Technology Research and Application Center, 42031, Konya, Turkey \\ ${ }^{3}$ Selçuk University, Directorate of Laser Induced Proton Therapy Application and Research Center, 42031, Konya, Turkey \\ ${ }^{4}$ Konya Necmettin Erbakan University, Department of Physics, Faculty of Ahmet Keleşoğlu Education, 42090, Konya, Turkey
}

\section{Highlights}

- ATP molecule has been investigated as theoretical in gas phase and aqueous solution.

- The first ten excited states of ATP molecule have been calculated using DFT and TD-DFT.

- HOMO, LUMO, HOMO-LUMO energy gap have been calculated in details.

- Chemical hardness, electronic chemical potential, electronegativity, electrophilicity investigated.

\section{Article Info}

Received:01 Dec 2020 Accepted:12 Mar 2021

\begin{tabular}{l} 
Keywords \\
\hline$A T P$ \\
$D F T$ \\
$T D-D F T$ \\
Absorption \\
Emission
\end{tabular}

\begin{abstract}
In this study, the conformational structure of the tetra-protonated Adenosine 5'triphosphate molecule has been investigated using Spartan 08 package program with the molecular dynamics method. Following the conformational analysis; geometry optimization, excited states, absorption and fluorescence (emission) spectra, molecular orbitals, chemical hardness, electronic chemical potential, electronegativity and electrophilicity indexes of the molecule were calculated by using density functional theory and time-dependent density functional theory method with B3LYP functional with $6-311+\mathrm{G}(\mathrm{d}, \mathrm{p})$ basis set. All calculations for the tetra-protonated ATP molecule have also been carried out both in the gas phase and in the aqueous solution and then results were compared with the experimental data reported in the literature.
\end{abstract}

\section{INTRODUCTION}

Deoxyribonucleic acid (DNA) is the name of biomolecule which carries genetic information of all living cells. It is a polynucleotide structure which is composed of various units such as nucleotides. All nucleotides consist of DNA bases (adenine, guanine, cytosine and thymine), a deoxyribose (sugar) and a phosphate group. They are named according to nucleobases for example, Adenosine 5'-triphosphate (ATP). DNA may have mutations when exposed to sunlight. These mutations, however, may be sometimes repaired; in some cases, these mutations can cause cancer. Cancer is may be a result of some photochemical and photophysical process. fluorescence process is one of these cancer-causing processes. Fluorescence behaviours can be investigated by examining the excited states. The excited states of nucleic acids have been studied and well understood.

DNA and RNA molecules have been extensively studied either theoretically or experimentally. However, these studies are still limited for well understanding the genetic information in DNA and RNA. The studies on solving these genetic codes have been carried out in the fields of physics, chemistry, biology and medicine $[1,2]$. ATP is one of the most important member of nucleotides family, since it is an universal energy source and an extracellular signalling intermediary in many biological processes, including DNA replications [3-5]. As well as ATP is stores and transports chemical energy 
within cells. Recent works show that ATP can be associated with some diseases such as parkinson, alzheimer and cancer [6, 7]. In order to better understand and interpret how these diseases relate to ATP molecule, it may be necessary to know the properties of the molecule in various environments and phases [8]. The binding process of ATP to proteins is a popular issue to study, so ATP molecule is an important examination for physical, chemical and biological properties in different environments and phases. However, the excited states of ATP molecule protonated with hydrogen atoms in gas phase and in aqueous solution have not been studied and reported in literature due to the knowledge of our group $[9,10]$.

The phosphate group in ATP molecule is negatively charged. These negative charges can be stabilized by hydrogen bonding that defines as corner stone of three-dimensional structure of molecules [10-12]. Van Outersterp et al. have suggested that the phosphate tail could be stabilized with hydrogen bonding [13]. These tails are usually protonated with metal cations [14], via water molecules and amino acid residues. [11, 13-18]. However, the phosphate tail of protonated ATP molecule has not been also completely examined as highlighted in literature due to the knowledge of our group $[13,19]$.

There are many experimental and theoretical studies on ATP molecule. Molecular structures of some ATP salts have been investigated by using X-ray diffraction (XRD) method. Herein, XRD data have shown that ATP molecule was folded towards to the phosphate group of the adenine base. [20, 21]. Hydrolysis process of ATP molecule was investigated theoretically adding magnesium ions and reported in literature [22]. Conformational study and spectral properties of ATP molecule protonated with $\mathrm{Mg}$ ions have been experimentally investigated using Nuclear Magnetic Resonance (NMR) method [23, 24]. On the other hand, doubly deprotonated ATP anions in gas phase and aqueous solution have been examined for structural properties of the adenine moiety by using infrared multiphoton dissociation spectroscopy [18], photo-electron spectroscopy [12], electrospray ionization mass spectrometry (ESIMS) [10] and collision-induced dissociation (CID) [13]. In theoretical studies, (ATP-2H)-1, (ATP-2H)2 [18], (H3PO4)-1 anions [10] and adenosine 5-diphosphate (ADP) have also been calculated by using Gaussian09 for quantum chemical calculations to determine its effect on nucleic base of phosphate tail and performed on infrared spectrum [13] absorption spectrum [18]. Burke and Schinle have suggested that negatively charged parts in phosphate groups can be stabilized by hydrogen bonds. Jones and Akola suggested that The ATP molecule can be investigated the effect of phosphate tail on nucleic base. Van Outersterp et al has examined the effect between the base and the phosphate tail by considering this suggestion either experimentally or theoretically. However, it has been reported that the phosphate tail was not completely protonated. The detail of excited states of nucleobases is quite inadequate [25]. Although the excited states have been studied for various molecules for example AMP [19, 25-27], the excited states are still not studied for ATP molecule.

The goal of present work is to investigate excited states theoretically, frontier molecular orbitals (FMOs), global reactivity descriptors and absorption and fluorescence (emission) spectra by stabilizing the phosphate tail of ATP molecule with hydrogen atom. Another important goal of present work is to contribute theoretically to mass spectroscopy studies for molecular weight and ordering of charged bio molecules. Theoretical calculations were carried out using TD-DFT and DFT methods at the B3LYP/6$31+\mathrm{G}(\mathrm{d}, \mathrm{p})$ level of theory. These calculations have been calculated in both of the gas phase and in aqueous solution. Then, the structural differences between the gas phase and aqueous solution have been examined and theoretical results obtained in this study have been compared with the experimental data available in literature.

\section{MATERIAL AND METHODS}

Quantum chemical calculations were carried out using Gaussian 09 and Spartan 08 software packages $[28,29]$. Spartan 08 program was used to establish possible stable conformations of titled molecules. These conformer structures were determined using molecular mechanical method as described in details elsewhere [30]. Later, the optimization of molecular structures, energies of excited states were carried 
out via DFT and TD-DFT methods using Gaussian 09 program [12]. The ground-state geometry (S0) of ATP was optimized using the B3LYP/6-31+G (d,p) level of theory.

Fluorescence (emission) calculations were carried out in seven steps. Step 1 was geometry optimization of molecules in the ground state. This calculations were performed at the B3LYP/6-31+G (d,p) level and by DFT method. Step 2 was obtaining the structure of the excited states of molecules. These steps were performed at the same level of theory, which was acquired by geometry optimization of molecules and used by TD-DFT method. Step 3 requires two consequential processes: firstly, the ground state calculation was carried out, specifying the Non Eq=Save choice, in order to store the information about non-equilibrium solvation based on the ground state. Secondly, the real state precise calculation was performed by reading in the requisite data to non-equilibrium solvation by using Non Eq=read choice, and then specifying the checkpoint file from Step 1. Step 4 was the excited states obtained in the last step, which were carried out geometry optimization that is interested in the geometry of excited state (S1). Step 5 frequency calculations in the excited state were performed because of this step runs a frequency calculation to approve which the geometry obtained in step 4 has a minimum energy. Step 6 was calculation of state specific equilibrium solvation of the excited state at its equilibrium geometry, writing out the solvation data for the next step via the nonequilibrium $=$ Save option., In step 7, the ground state energy was calculated from a non-equilibrium solvation computation in solution by using the exited state optimized geometry. The Polarizable Continuum Model (PCM) of the self-consistent reaction field (SCRF) theory is one of the best known approaches to determine states (either ground or excited) in solution [31, 32]. Herein the absorption and Fluorescence (emission) energies were computed using Gaussian 09. Water was used as a solvent in our calculations.

\section{THE RESEARCH FINDINGS AND DISCUSSION}

\subsection{Optimized Geometry}

The three-dimensional structure of neutral ATP molecule is shown in Figure 1. When examining the properties of a molecule, the most important step is the correct determination of the three-dimensional structure of a molecule. Because the correct interpretation and understanding of the molecular properties depends on the correct determination of this step. Firstly, three-dimensional structure of ATP molecule was created using Gaussian 09 programs. Then, conformer analysis of molecule was carried out by using Spartan 08 program as described in details elsewhere [30]. As a result of conformational analysis, 100 different conformer structures were found by using Spartan 08 program and molecular force field method. Since results have been obtained with a study using the same program and method in literature [10], these structures have not been given in this study. However, the each structure was optimized using B3LYP / 6-311 + G (d, p) level of DFT method. Thus, the most stable structure of title molecule was obtained and this structure was given in Figure 1. (Full structure for the most stable conformer may be available in supplementary materials). The results obtained work are quite compatible with theoretical calculations obtained in literature [10]. The crystal structure of molecule was investigated experimentally elsewhere [33]. The structure of ATP molecule obtained has been given in Figure 1 which shows that the phosphate group is bent towards the adenine base. It is quite compatible with the previously obtained structure [18]. 


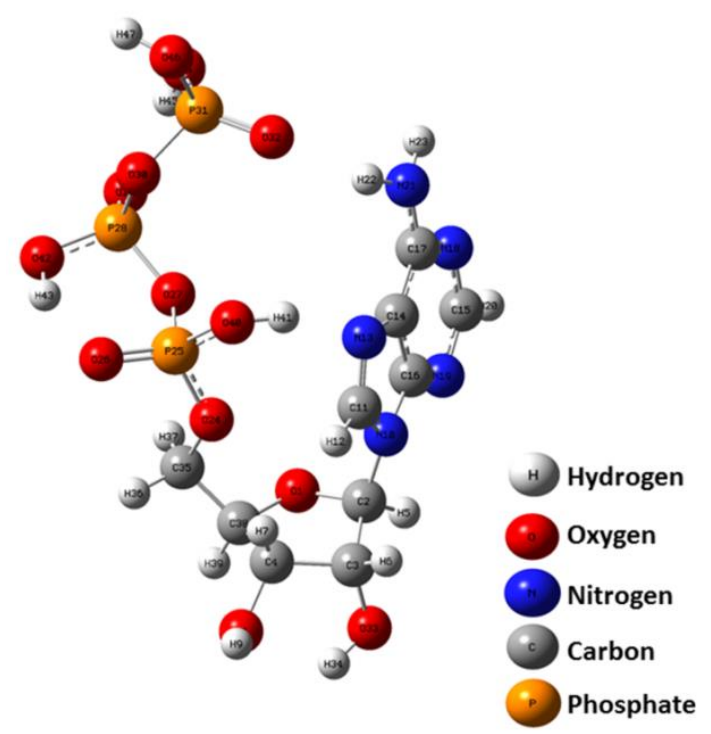

Figure 1. The most stable and the three-dimensional structure of the ATP molecule. The most stable structure of the molecule is a structure with minimum energy

The energy, dipole moment and polarity values of the title molecule were obtained as $72581.05 \mathrm{eV}, 5.98$ Debye and $6865.96 \mathrm{eV}$, respectively. Bond lengths, bond angles and dihedral angles of obtained structure were also estimated by using DFT method with the $6-311+\mathrm{G}(\mathrm{d}, \mathrm{p})$ basis set. The selected value is presented in Table 1 and obtained results were compared with literature [18, 33].

DFT method was used to optimize the structure of the ground state ATP molecule in gas phase and in aqueous solution. However, TDDFT method was used to optimize excited structures. Some selected bond lengths, bond angles and dihedral angles for the excited state structures of ATP molecule in gas phase and aqueous solution are listed in Table 1. the optimized structures in ground state and excited state are shown in Figure 2. 

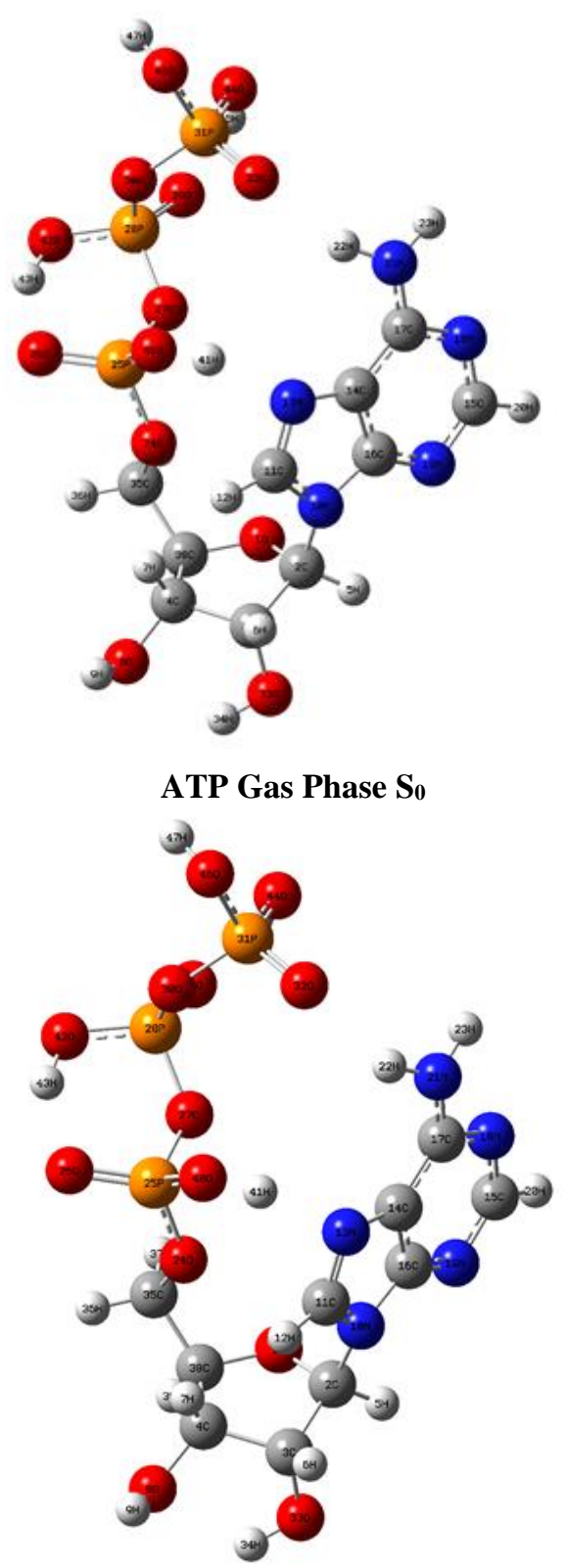

ATP Solution $\mathbf{S}_{\mathbf{0}}$

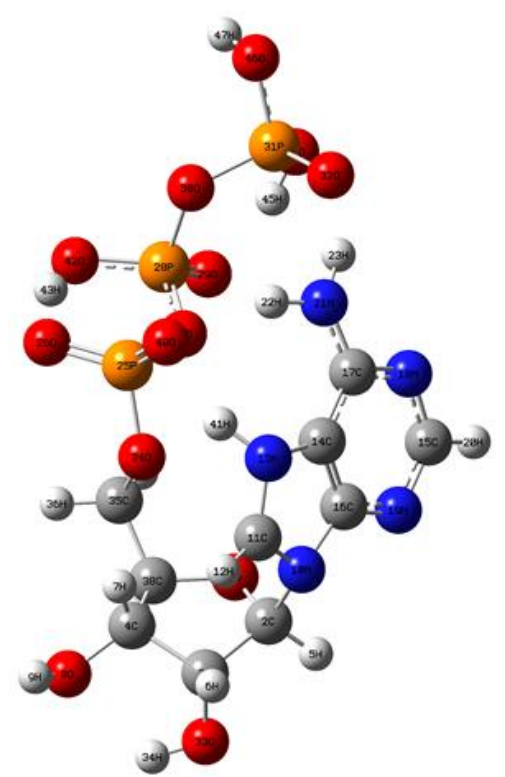

ATP Gas Phase $\mathbf{S}_{1}$

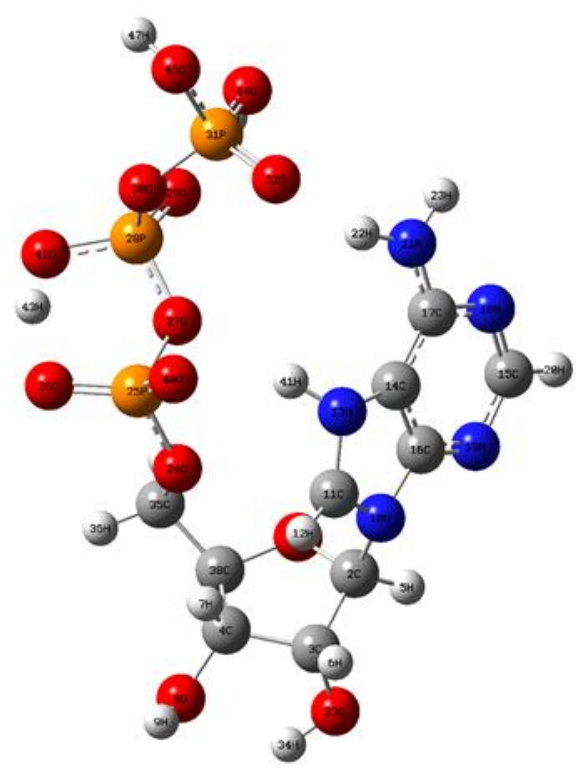

ATP Solution $\mathbf{S}_{\mathbf{1}}$

Figure 2. Optimized structures of ATP molecule in gas phase and aqueous solution ( $S O$ is the ground state geometry, $\mathrm{S} 1$ is the excited state geometry)

The theoretical and experimental studies support that the phosphate group of obtained ATP molecule structure is bent towards adenine [34-36]. The obtained theoretical results and XRD results given in literature have been compatible. However, the study observed is little different for some obtained bond lengths, bond angles and dihedral angles. Because the hydrogen atoms added to stabilize the molecule or used different method may have been caused to these differences. Theoretical results obtained from the stabilized ATP molecule will provide useful theoretical data for future experimental studies. 
Table 1. The table gives a comparison of parameters of the molecule due to the bond lengths, bond angles and dihedral angles selected of ATP molecule in the gas phase and the aqueous solution based on the theoretical and $x$-ray diffraction data in the literature. The ground-state geometry (SO), the geometry of excited state (S1) $a=$ [34], $b=$ [35], $c=$ [36]

\begin{tabular}{|c|c|c|c|c|c|c|}
\hline $\begin{array}{c}\text { Bond Length } \\
\AA\end{array}$ & $\begin{array}{c}\text { Gas phase } \\
\left(\mathbf{S}_{0}\right)\end{array}$ & $\begin{array}{c}\text { Solution } \\
\left(\mathbf{S}_{\mathbf{0}}\right)\end{array}$ & $\begin{array}{c}\text { Gas phase } \\
\left(\mathrm{S}_{1}\right)\end{array}$ & $\begin{array}{c}\text { Solution } \\
\left(\mathbf{S}_{1}\right)\end{array}$ & $\begin{array}{c}\text { Theoretical } \\
\text { Ref. }\end{array}$ & $\begin{array}{c}\text { Experimental } \\
\text { Ref. }\end{array}$ \\
\hline P31-O46 & 1.59 & 1.59 & 1.59 & 1.59 & & $1.43^{\mathrm{a}}(1.44)^{\mathrm{b}}$ \\
\hline P31-032 & 1.47 & 1.48 & 1.48 & 1.48 & & $1.55^{\mathrm{a}}(1.47)^{\mathrm{b}}$ \\
\hline P31-O44 & 1.59 & 1.59 & 1.58 & 1.59 & & $1.43^{\mathrm{a}}(1.58)^{\mathrm{b}}$ \\
\hline P31-O30 & 1.65 & 1.65 & 1.64 & 1.63 & $1.68^{\mathrm{c}}$ & $1.53^{\mathrm{a}}(1.59)^{\mathrm{b}}$ \\
\hline P28-O30 & 1.62 & 1.62 & 1.64 & 1.64 & $1.61^{\mathrm{c}}$ & $1.70^{\mathrm{a}}$ \\
\hline $\mathrm{P} 28-\mathrm{O} 29$ & 1.48 & 1.48 & 1.49 & 1.49 & & $1.46^{\mathrm{a}}$ \\
\hline $\mathrm{P} 28-\mathrm{O} 42$ & 1.57 & 1.57 & 1.56 & 1.57 & & $1.60^{\mathrm{a}}$ \\
\hline P28-027 & 1.62 & 1.61 & 1.60 & 1.59 & $1.63^{\mathrm{c}}$ & $1.70^{\mathrm{a}}(1.59)^{\mathrm{b}}$ \\
\hline $\mathrm{P} 25-\mathrm{O} 27$ & 1.65 & 1.65 & 1.70 & 1.71 & $1.62^{\mathrm{c}}$ & $1.54^{\mathrm{a}}$ \\
\hline $\mathrm{P} 25-\mathrm{O} 26$ & 1.49 & 1.49 & 1.51 & 1.52 & & $1.52^{\mathrm{a}}(1.48)^{\mathrm{b}}$ \\
\hline P25-O40 & 1.56 & 1.56 & 1.50 & 1.49 & & $1.58^{\mathrm{a}}(149)^{\mathrm{b}}$ \\
\hline $\mathrm{P} 25-\mathrm{O} 24$ & 1.59 & 1.58 & 1.62 & 1.60 & & $1.46^{\mathrm{a}}(1.63)^{\mathrm{b}}$ \\
\hline \multicolumn{7}{|l|}{$\begin{array}{l}\text { Bond Angles } \\
\text { (in degree) }\end{array}$} \\
\hline $\mathrm{O} 40-\mathrm{P} 25-\mathrm{O} 26$ & 116.2 & 115.1 & 123.8 & 122.7 & & $119^{\mathrm{a}}(118)^{\mathrm{b}}$ \\
\hline O40-P25-O27 & 106.8 & 105.9 & 107.1 & 107.3 & & $108^{\mathrm{a}}(110)^{\mathrm{b}}$ \\
\hline O40-P25-O24 & 103.6 & 104.3 & 105.7 & 107.0 & & $107^{\mathrm{a}}(110)^{\mathrm{b}}$ \\
\hline O26-P25-O27 & 109.3 & 108.9 & 105.3 & 104.0 & & $101^{\mathrm{a}}(109)^{\mathrm{b}}$ \\
\hline $\mathrm{O} 26-\mathrm{P} 25-\mathrm{O} 24$ & 117.0 & 118.6 & 112.5 & 113.3 & & $116^{\mathrm{a}}$ \\
\hline $\mathrm{O} 27-\mathrm{P} 25-\mathrm{O} 24$ & 102.3 & 102.4 & 99.4 & 99.5 & & $105^{\mathrm{a}}(99)^{\mathrm{b}}$ \\
\hline O27-P28-O42 & 105.8 & 106.3 & 106.9 & 106.8 & & $102^{\mathrm{a}}$ \\
\hline $\mathrm{O} 27-\mathrm{P} 28-\mathrm{O} 29$ & 112.3 & 112.4 & 113 & 113.7 & & $104^{\mathrm{a}}$ \\
\hline O27-P28-O30 & 103.8 & 103.9 & 103.9 & 104.4 & & $100^{\mathrm{a}}$ \\
\hline O42-P28-O29 & 117.7 & 116.8 & 118.2 & 117.5 & & $121^{\mathrm{a}}$ \\
\hline O42-P28-O30 & 104.1 & 104.4 & 104.3 & 103.6 & & $113^{\mathrm{a}}$ \\
\hline $\mathrm{O} 29-\mathrm{P} 28-\mathrm{O} 30$ & 111.7 & 111.6 & 109.0 & 109.3 & & $114^{\mathrm{a}}$ \\
\hline O30-P31-032 & 112.8 & 112.9 & 112.9 & 113.5 & & $99^{\mathrm{a}}(106)^{\mathrm{b}}$ \\
\hline O30-P31-O44 & 103.1 & 104.2 & 104.3 & 104.5 & & $111^{\mathrm{a}}$ \\
\hline O30-P31-O46 & 102.6 & 103.1 & 103.5 & 103.8 & & $109^{\mathrm{a}}(112)^{\mathrm{b}}$ \\
\hline O32-P31-O44 & 119.2 & 117.6 & 118.3 & 116.8 & & $109^{\mathrm{a}}(107)^{\mathrm{b}}$ \\
\hline O44-P31-O46 & 104.4 & 106.7 & 105.3 & 107 & & $111^{\mathrm{a}}(109)^{\mathrm{b}}$ \\
\hline P25-027-P28 & 124.7 & 125.6 & 127.4 & 124.0 & & $118^{\mathrm{a}}$ \\
\hline P28-O30-P31 & 125.5 & 129.5 & 126.1 & 127.3 & & $136^{\mathrm{a}}(131)^{\mathrm{b}}$ \\
\hline C35-O24-P25 & 127 & 127.2 & 123.1 & 126.9 & & $139^{\mathrm{a}}(121)^{\mathrm{b}}$ \\
\hline \multicolumn{7}{|l|}{$\begin{array}{c}\begin{array}{l}\text { Dihedral Angles } \\
\text { (in degree) }\end{array} \\
\end{array}$} \\
\hline C35-O24-P25-O27 & 110.4 & 105.9 & 116.4 & 110 & & $135^{\mathrm{a}}$ \\
\hline O24-P25-O27-P28 & 155.3 & 156.2 & 139 & 153.7 & & $153^{\mathrm{a}}$ \\
\hline O27-P28-O30-P31 & 87.1 & 91.5 & 93 & 86 & & $83^{\mathrm{a}}$ \\
\hline
\end{tabular}

For easier comparison of the results obtained in Table 1 and XRD results, two digits after the point in bond lengths and four digits in bond and dihedral angles are not taken into account.

\subsection{Excited State}

The electronically excited state is any quantum state of atomic or molecular system with higher energy level above ground state. The molecular orbital analyses are important to study the nature of electronically excited states. The examination of the photo physical properties of a molecular system depends on the electronically excited state processes that occur after absorbing photons. HOMO and LUMO are types of molecular orbitals. The difference between these orbitals is known as HOMO LUMO energy gap and is an important parameter for determining electrical properties of molecules. the 
excitation energy, wavelength, oscillator strengths and major contributions in gas phase and aqueous solution of title molecule were calculated using TD-DFT method and TD-DFT PCM method with the 6-31+G (d,p) basis set. These values are shown in Table 2 for gas phase and shown in Table 3 for aqueous solution. The first excited state in gas phase corresponds to $268.6 \mathrm{~nm}$. This value was calculated to be $259.5 \mathrm{~nm}$ in aqueous solution. The oscillator strength obtained in both cases were calculated as 0.15 and 0.2444 , respectively. The major contributions for both environments were observed in HOMO $\rightarrow$ LUMO transition corresponding to S1 state. The highest value obtained in the gas phase is obtained in the transition from HOMO to LUMO+2 (98\%). However, the highest values obtained in aqueous solution were obtained in transitions both from HOMO to LUMO+1 and from HOMO-1 to LUMO+2 (96\%). The values obtained for each excited state are listed in Tables 2 and Table 3.

Table 2. Time-dependent density functional theory calculated excitation energies $(\Delta E)$, wavelength $(\lambda)$, oscillate strengths $(f)$ and major contributions configurations for the low-lying excited states of Gas phase ATP molecule. HOMO and LUMO are types of molecular orbitals ( $H=$ the highest occupied molecular orbital and $L=$ the lowest lying unoccupied molecular orbital)

\begin{tabular}{|c|c|c|c|c|}
\hline \multicolumn{5}{|c|}{ Calculated Values } \\
\hline Excited State & $\begin{array}{c}\text { Excitation } \\
\text { energies }\left(\mathbf{c m}^{-1}\right) \\
(\Delta \mathbf{E})\end{array}$ & $\begin{array}{c}\text { Wavelength } \\
(\mathbf{n m}) \\
(\lambda)\end{array}$ & $\begin{array}{c}\text { Oscillator } \\
\text { strengths } \\
(f)\end{array}$ & Major Contributions \\
\hline $\mathrm{S}_{1}$ & 37224.3 & 268.6 & 0.15 & HOMO->LUMO (89\%) \\
\hline $\mathrm{S}_{2}$ & 38439 & 260.1 & 0.0103 & HOMO->L+1 (93\%) \\
\hline $\mathrm{S}_{3}$ & 39168.1 & 255.3 & 0.0026 & H-1->LUMO (78\%), H-1->L+1 (15\%) \\
\hline $\mathrm{S}_{4}$ & 40653.8 & 245.9 & 0.0049 & HOMO->L+2 (98\%) \\
\hline $\mathrm{S}_{5}$ & 41344.2 & 241.8 & 0.0394 & H-2->LUMO (14\%), HOMO->L+3 (69\%) \\
\hline $\mathrm{S}_{6}$ & 43772.8 & 228.4 & 0.0026 & H-1->L+3 (89\%) \\
\hline $\mathrm{S}_{7}$ & 45306 & 220.7 & 0.0236 & HOMO->L+4 (77\%), HOMO->L+5 (15\%) \\
\hline $\mathrm{S}_{8}$ & 45654.5 & 219 & 0.0007 & H-1->LUMO (15\%), H-1->L+1 (77\%) \\
\hline $\mathrm{S}_{9}$ & 46186.8 & 216.5 & 0.012 & HOMO->L+4 (17\%), HOMO->L+5 (80\%) \\
\hline $\mathrm{S}_{10}$ & 47276.5 & 211.5 & 0.0429 & HOMO->L+6 (69\%) \\
\hline
\end{tabular}

Table 3. Time-dependent density functional theory calculated excitation energies $(\Delta E)$, wavelength $(\lambda)$, oscillate strengths $(f)$ and major contributions for the low-lying excited states of ATP molecule in aqueous solution. HOMO and LUMO are types of molecular orbitals (H= the highest occupied molecular orbital and $L=$ the lowest lying unoccupied molecular orbital)

\begin{tabular}{|c|c|c|c|c|}
\hline \multicolumn{5}{|c|}{ Calculated Values } \\
\hline Excited State & $\begin{array}{c}\text { Excitation } \\
\text { energies }\left(\mathbf{c m}^{-1}\right) \\
(\Delta E)\end{array}$ & $\begin{array}{c}\text { Wavelength } \\
\text { (nm) } \\
(\lambda)\end{array}$ & $\begin{array}{c}\text { Oscillator } \\
\text { strengths } \\
(f)\end{array}$ & Major Contributions \\
\hline $\mathrm{S}_{1}$ & 38535 & 259.5 & 0.2444 & HOMO->LUMO $(89 \%)$ \\
\hline $\mathrm{S}_{2}$ & 40703.8 & 245.6 & 0.0022 & H-1->LUMO (94\%) \\
\hline $\mathrm{S}_{3}$ & 41107.1 & 243.2 & 0.0565 & $\begin{array}{c}\text { H-2->LUMO }(15 \%), \text { HOMO->L+2 } \\
(74 \%)\end{array}$ \\
\hline $\mathrm{S}_{4}$ & 42366.1 & 236 & 0.0073 & HOMO->L+1 (96\%) \\
\hline $\mathrm{S}_{5}$ & 44768.9 & 223.3 & 0.0026 & $\mathrm{H}-1->\mathrm{L}+2(96 \%)$ \\
\hline $\mathrm{S}_{6}$ & 46998.2 & 212.7 & 0.0301 & $\begin{array}{c}\text { HOMO->L+3 }(36 \%), \text { HOMO->L+4 } \\
(54 \%)\end{array}$ \\
\hline $\mathrm{S}_{7}$ & 47115.2 & 212.2 & 0.0135 & $\begin{array}{c}\text { HOMO->L+3 }(61 \%), \text { HOMO->L+4 } \\
(30 \%)\end{array}$ \\
\hline
\end{tabular}




\begin{tabular}{|c|c|c|c|c|}
\hline $\mathrm{S}_{8}$ & 47859.6 & 208.9 & 0.1957 & $\begin{array}{c}\text { H-4->LUMO (12\%), H-2->LUMO (67\%) } \\
\text { HOMO->L+2 (10\%) }\end{array}$ \\
\hline $\mathrm{S}_{9}$ & 48740.4 & 205.1 & 0.0115 & $\begin{array}{c}\text { H-6->LUMO (16\%), HOMO->L+6 } \\
(68 \%)\end{array}$ \\
\hline $\mathrm{S}_{10}$ & 48848.4 & 204.7 & 0.0093 & $\begin{array}{c}\text { H-6->LUMO (60\%), HOMO->L+6 } \\
(17 \%)\end{array}$ \\
\hline
\end{tabular}

The frontier molecular orbital (FMO) analysis is highly important to understand the nature of orbital taking part in chemical reactions and to draw electronic spectra. The chemical stability of a molecule can be defined by the energy difference between HOMOs and LUMOS levels [3]. FMO energy levels of gas phase ATP molecule were computed using TD-DFT method at B3LYP/631+G (d,p) level of theory. The B3LYP used in this work can be underestimates the HOMO-LUMO energy gaps and excited-state energies but the CAM-B3LYP can be a way to correct for this deficiency as described in the recent Works [37-39]. The molecular orbital energy surfaces of several important FMOs are given in Figure 3. HOMO and LUMO values were found as $-8.41 \mathrm{eV}$ and $-4.22 \mathrm{eV}$ respectively. The energy band gap between HOMO and LUMO was calculated as $4.19 \mathrm{eV}$, whereas the energy gap between other important transitions from HOMO to LUMO+1, from HOMO to LUMO+2 and HOMO-1 to LUMO were determined to be $4.69 \mathrm{eV}, 5.46 \mathrm{eV}$ and $4.81 \mathrm{eV}$, respectively. As shown in Figure 4, the HOMO, LUMO and the HOMO-LUMO energy gap values of ATP molecule in aqueous solution are calculated to be $(-8.87 \mathrm{eV}),-4.79 \mathrm{eV}$ and $4.08 \mathrm{eV}$, respectively. Other important transitions in aqueous solution, from HOMO-1 to LUMO, from HOMO to LUMO+1 and HOMO-1 to LUMO+2 were determined to be $4.77 \mathrm{eV}, 4.55 \mathrm{eV}$ and $6.01 \mathrm{eV}$, respectively.
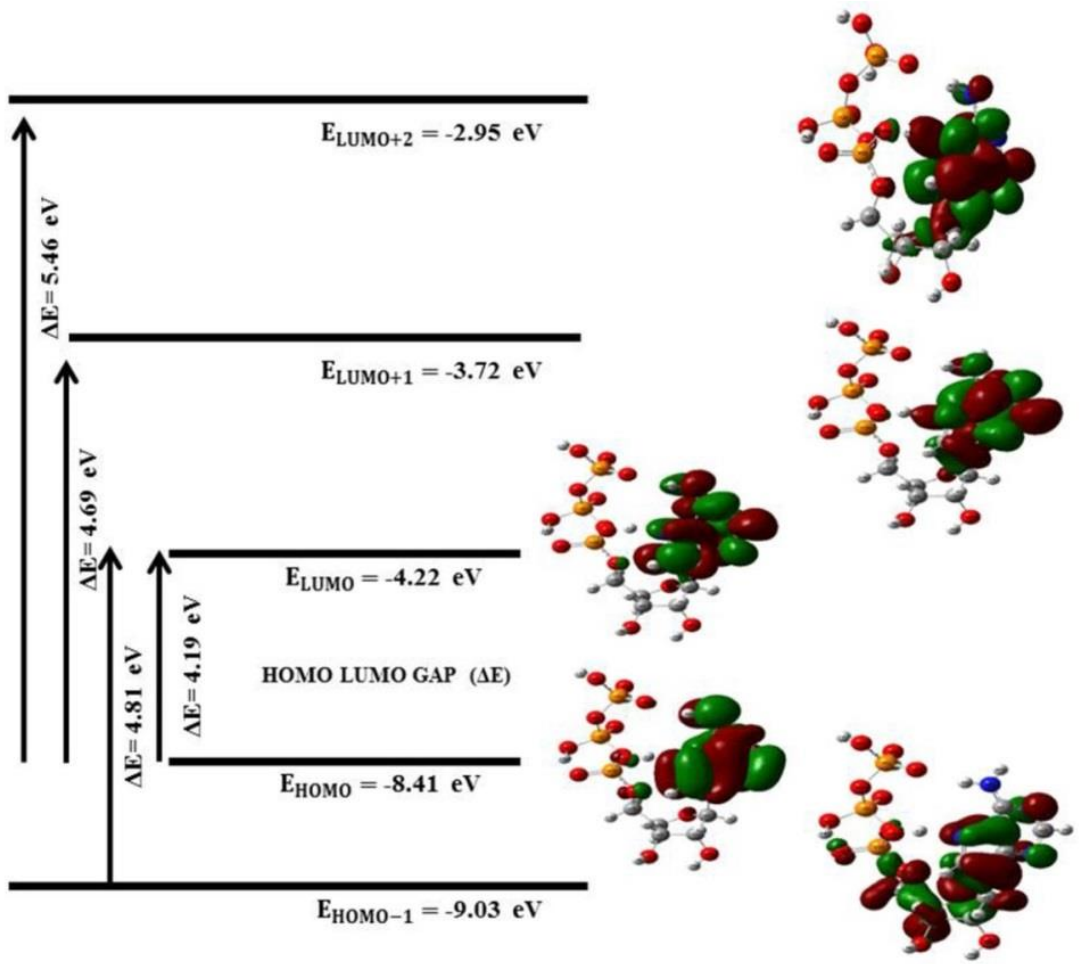

Figure 3. Selected frontier molecular orbitals of Gas phase ATP molecule with the energy gaps. HOMO and LUMO are types of molecular orbitals (HOMO=Highest occupied molecular orbital and $L U M O=$ Lowest unoccupied molecular orbital maps. The energy gap between HOMO and LUMO has been a critical parameter to determine the molecular electrical properties 
The most likely transition in gas phase is from HOMO to LUMO + 2, and the oscillator value of this transition was obtained as 0.00049 . But these transitions in aqueous solution were obtained as from HOMO to LUMO+1 and HOMO-1 to LUMO+2 and the oscillator strengths values of these transitions have obtained as 0.0073 and 0.0026 , respectively.
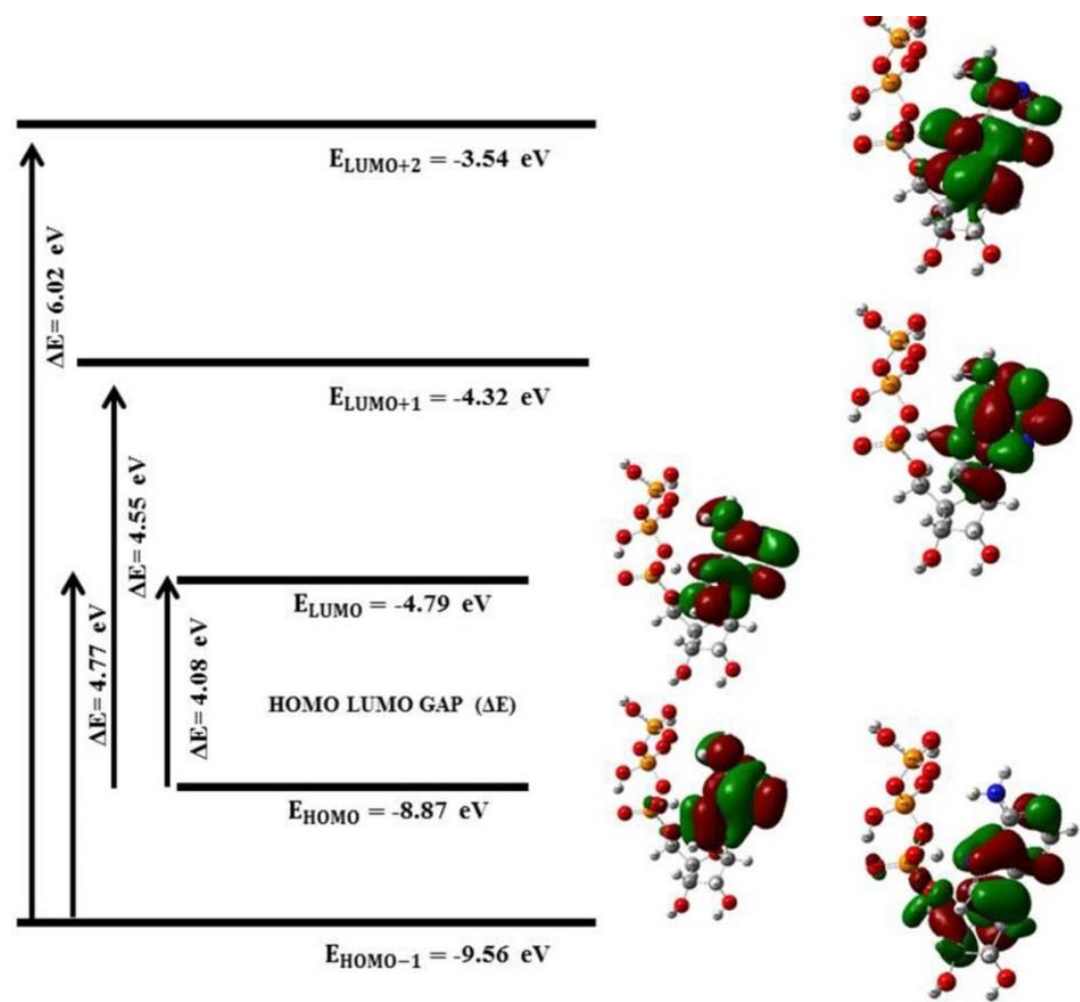

Figure 4. Selected frontier molecular orbitals of ATP molecule in aqueous solution with the energy gaps. HOMO and LUMO are types of molecular orbitals (HOMO=Highest occupied molecular orbital and $L U M O=$ Lowest unoccupied molecular orbital maps. The energy gap between HOMO and LUMO has been a critical parameter to determine the molecular electrical properties

FMOs for both environments are generally localized on adenine base. However, in case of HOMO-1, the frontier molecular orbitals are localized in both sugar and base groups.

\subsection{Global Reactivity Descriptors}

The Global reactivity descriptors show characteristics of a molecule. Thanks to FMOs, the Global reactivity descriptors such as, chemical hardness $(\eta)$, electronic chemical potential $(\mu)$, electronegativity $(\chi)$ and electrophilicity index $(\omega)$ have been evaluated. The chemical hardness is an important parameter when the stability and reactivity of a molecule system are examined. This parameter was calculated by using $\eta=(E L U M O-E H O M O) / 2$ [40]. The chemical hardness in gas phase was found to be $2.09 \mathrm{eV}$ and the chemical hardness value in aqueous solution was lower than that in gas phase and was calculated to be $02.04 \mathrm{eV}$. As a result, we can say that is better in gas phase when chemical hardness is compared for both environments. The negative electronegativity of a molecule system can be defined as an electronic chemical potential and calculated by using $\mu=($ EHOMO+ELUMO $) / 2$ value [41]. The electronegativity can be defined as measurement in which an atom tends to attract a shared pair of electrons and it can be calculated by equation $\chi=-(\mathrm{EHOMO}+\mathrm{ELUMO}) / 2$ [42]. Electrophilicity index is calculated by $\omega 2=\mu 2 / 2 \eta$ and it is defined as a measure of tendency of a molecular system to accept electrons [43]. The electrophilicity index is reliable indicator of reactivity and stabilization energy [44, 45]. These global reactivity values are given in Table 4. 
Table 4. The Chemical reactivity parameters obtained in gas phase and aqueous solution of ATP molecule. These parameters are the global reactivity descriptors in terms of molecular stability

\begin{tabular}{|c|c|c|}
\hline \multicolumn{3}{|c|}{ Calculated Values } \\
\hline Chemical hardness & Gas phase & Aqueous solution \\
\hline Electronic chemical potential & $1.90 \mathrm{eV}$ & $1.90 \mathrm{eV}$ \\
\hline Electronegativity & $-6.25 \mathrm{eV}$ & $-6.80 \mathrm{eV}$ \\
\hline Electrophilicity index & $6.25 \mathrm{eV}$ & $6.80 \mathrm{eV}$ \\
\hline
\end{tabular}

\subsection{Spectroscopic Results}

UV-Vis absorption spectra of various large molecules have been successfully calculated by TD-DFT method by using quantum chemical calculations which have been reported already in literature [46, 47]. In this study, TD-DFT has been used to predict absorption and emission wavelengths in both gas phase and aqueous solution for neutral ATP molecule. Electronically excited states of a molecular system are an important parameter for understanding and interpreting the process the system goes through after absorption of light. This process also forms a basis for photon-matter interaction studies. The electronically excited state is transition from the ground state to the excited state after absorption of one or more photons by molecule. Absorption spectra were determined using TDDFT procedure on the ground state geometries of molecule. Emission spectra were determined using the same procedure in a transition period from the excited to the ground state. The calculation of these processes is explained in detail in the material and method sections. The shapes and structural parameters of the structures obtained are presented in Figure 2 and Table 1, respectively. The optical absorption spectra of isolated DNA base and base pairs were calculated using TD-DFT method [48].

Examination of absorption and fluorescence (emission) spectra can be difficult to handle without any help of quantum chemical calculations. In this work, UV-Vis absorption and fluorescence (emission) spectra in aqueous solution have been calculated using TD-DFT PCM B3LYP/6-31+G (d,p) level. These processes in gas phase was obtained using TD-DFT B3LYP/6-31+G (d,p) level. These methods provide some reliable results when calculation of absorption and fluorescence (emission) [49]. Figure 5 presents the absorption and fluorescence (emission) spectra of title molecule. The absorption and emission wavelengths obtained experimentally from literature are quite compatible with the results obtained in our theoretical study $[14,50,51]$.

UV-Vis spectrum has a very important tool to investigate the nature of the electronic structure in the excited state of molecules. In this study, when UV-Vis absorption spectrum obtained in the ground state are compared with theoretical data calculated in gas phase and aqueous solution, the results are seen that do not change between $260 \mathrm{~nm}$ and $300 \mathrm{~nm}$. However, this situation changes between $200 \mathrm{~nm}$ and 250 $\mathrm{nm}$. This change was obtained while examining the process in aqueous solution as expected. UV-Vis absorption spectrum obtained from quantum mechanical calculations showed that the electronic transition in gas phase occurred at wavelength of $268.65 \mathrm{~nm}$ and oscillator strengths corresponding to this electronic transition was 0.15 . Since ATP molecule has an aromatic structure, the maximum value of the absorption spectra is expected to be $260 \mathrm{~nm}$ as described in literature [51]. In aqueous solution, the electronic transition was calculated at wavelength of $259.50 \mathrm{~nm}$ and oscillator strength was calculated as 0.2444 . As we expected, it was observed that the absorption spectra shifted towards to blue due to aqueous solution effect. UV-Vis absorption spectrum was defined to be based on transitions from HOMOs to LUMOs. We have noticed that the absorption intensity in aqueous solution was decreased according to in gas phase; this reduction can changed depending on polarity of used solvent [52]. 


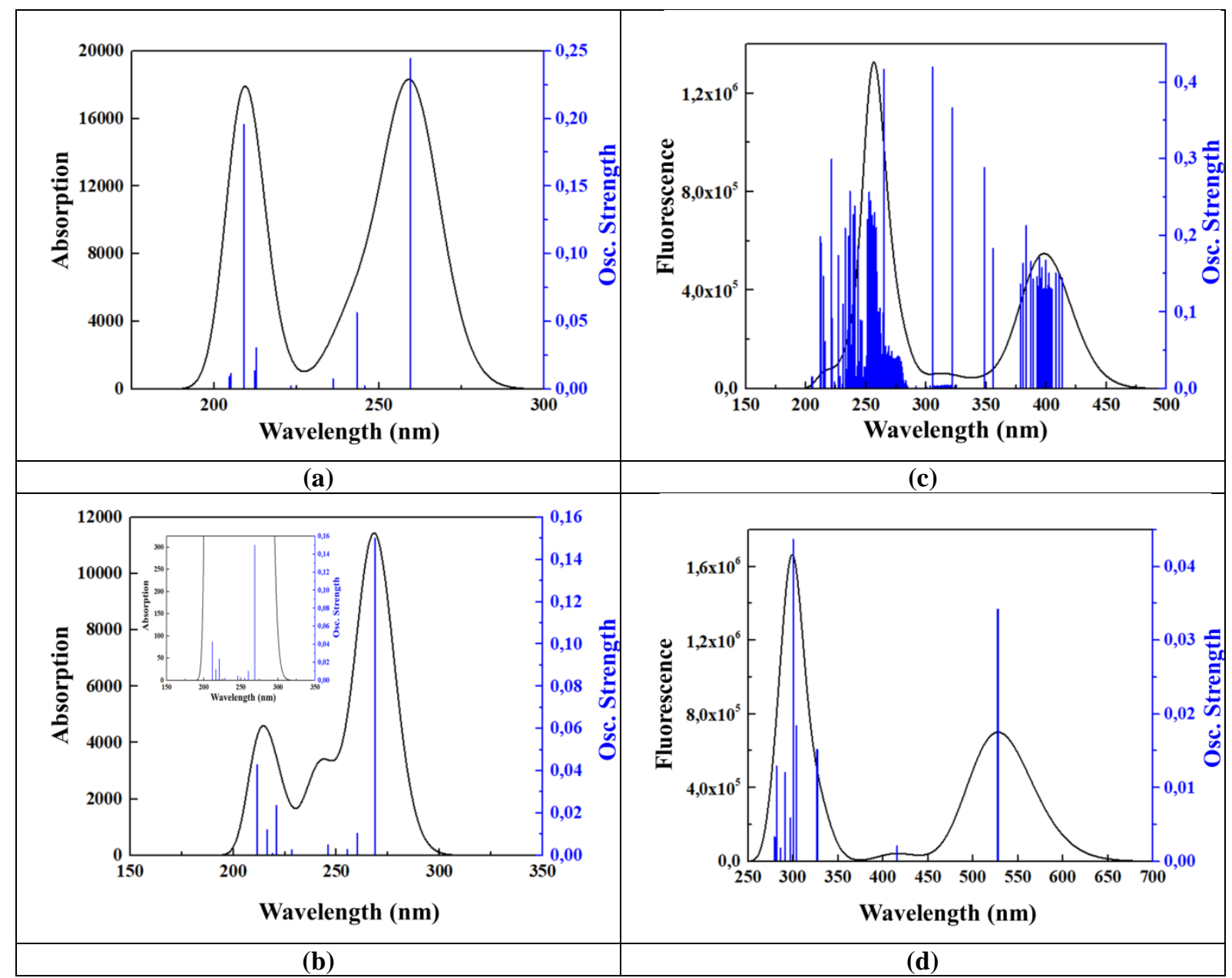

Figure 5. The UV-Vis absorption spectra of neutral ATP molecule a) in the aqueous solution and b) in the gas phase, the fluorescence (emission) spectra of neutral ATP molecule c) in the aqueous solution and $d)$ in the gas phase

Fluorescence (emission) spectrum has been obtained from optimized structure in the first excited state and these structures are given in Table 1. The fluorescence (Emission) process is defined as transitions from LUMO to HOMO. In some recent works given by Carrasquilla et.al. and Cervantes-Navarro et.al. the absorption and emission processes for different molecules were calculated by using the same methods [52,53]. Fluorescence (emission) spectrum of ATP molecule was experimentally obtained by Amat et.al. using a spectrofluorometer and the spectra were analysed by using Felix analysis mode software [15]. In this experimental study, the emission wavelength in aqueous solution was found to be in the range between 310 and $500 \mathrm{~nm}$ when excited by a laser light at $260 \mathrm{~nm}$ wavelength [14]. In another experimental study, this transition was obtained at $335 \mathrm{~nm}$ wavelength [54]. In this study, the emission wavelength aqueous solution was theoretically obtained as $399 \mathrm{~nm}$ and oscillator strength to be 0.1284 . The emission wavelength and oscillator strength in gas phase were calculated as $300 \mathrm{~nm}$ and 0.0434 , respectively. It is obvious that the theoretical and experimental results are in good agreement with each other $[15,54]$.

\section{CONCLUSIONS}

ATP is an important molecule for many biological processes. In this study, the DFT and TD-DFT PCM methods were used to study the physical properties of ATP molecule. The molecular structure, conformational analyses, electronic and chemical properties of the tetra-protonated ATP molecule were discussed theoretically for both in gas phase and in aqueous solution. Three-dimensional structure of ATP molecule was found that the phosphate group is bent towards the adenine base. This result is quite compatible with data in literature. Also, the results shows that the aqueous solution clearly affects the 
structural properties, frontier molecular orbitals (FMOs) as well as absorption and fluorescence (emission) spectra. This can be due to hydrogen atoms attached to phosphate group to stabilize the molecular structure. Due to the lack of experimental values on geometries of neutral ATP molecule either in gas phase or aqueous solution, the theoretical data obtained in this work were only compared with the current values in literature. From the physical point of view, determination of the excited states of ATP molecule can contribute to the development of biomedical technology. Results obtained in this study can be provided a useful theoretical data for future experimental studies for mass spectroscopy studies for molecular weight of charged bio molecules.

\section{CONFLICTS OF INTEREST}

No conflict of interest was declared by the authors.

\section{REFERENCES}

[1] Nir, E., Imhof, P., Kleinermanns, K., de Vries, M.S., "REMPI Spectroscopy of Laser Desorbed Guanosines", Journal of the American Chemical Society, 122(33): 8091-8092, (2000).

[2] Wu, R. R., Yang, B., Berden, G., Oomens, J., Rodgers, M. T., "Gas-phase conformations and energetics of protonated 2'-deoxyadenosine and adenosine: IRMPD action spectroscopy and theoretical studies", The Journal of Physical Chemistry B, 119(7): 2795-2805, (2015).

[3] Abraham, E. H., Okunieff, P., Scala, S., Vos, P., Oosterveld, M. J., Chen, A.Y., Shrivastav, B., "Cystic fibrosis transmembrane conductance regulator and adenosine triphosphate", Science, 275(5304): 1324-1326, (1997).

[4] Newman, E. A., Zahs, K. R., "Calcium waves in retinal glial cells", Science, 275(5301): 844-847, (1997).

[5] Szewczyk, A., Pikula, S., "Adenosine 5'-triphosphate: an intracellular metabolic messenger", Biochim Biophys Acta, 1365(3): 333-353, (1998).

[6] Bush, K. T., Keller, S. H., Nigam, S. K., "Genesis and reversal of the ischemic phenotype in epithelial cells", The Journal of Clinical Investigation, 106(5): 621-626, (2000).

[7] Ma, C., Chen, H., Han, R., He, H., Zeng, W., "Fluorescence detection of adenosine triphosphate using smart probe", Analytical Biochemistry, 429(1): 8-10, (2012).

[8] Neelakandan, P. P., Hariharan, M., Ramaiah, D., "Synthesis of a novel cyclic donor-acceptor conjugate for selective recognition of ATP", Organic Letters, 7(26): 5765-5768, (2005).

[9] Crespo-Hernández, C. E., Cohen, B., Hare, P. M., Kohler, B., "Ultrafast excited-state dynamics in nucleic acids", Chemical Reviews, 104(4): 1977-2020, (2004).

[10] Burke, R. M., Pearce, J. K., Boxford, W. E., Bruckmann, A., Dessent, C. E., "Stabilization of Excess Charge in Isolated Adenosine 5 '-Triphosphate and Adenosine 5 "Diphosphate Multiply and Singly Charged Anions”, The Journal of Physical Chemistry A, 109(43): 9775-9785, (2005).

[11] Sigel, H., Griesser, R., "Nucleoside 5'-triphosphates: self-association, acid-base, and metal ionbinding properties in solution", Chemical Society Reviews, 34(10): 875-900, (2005).

[12] Schinle, F., Crider, P. E., Vonderach, M., Weis, P., Hampe, O., Kappes, M. M., "Spectroscopic and theoretical investigations of adenosine 5'-diphosphate and adenosine 5'-triphosphate dianions in the gas phase", Physical Chemistry Chemical Physics, 15(18): 6640-6650, (2013). 
[13] Van Outersterp, R. E., Martens, J., Berden, G., Steill, J. D., Oomens, J., Rijs, A. M., "Structural characterization of nucleotide 5'-triphosphates by infrared ion spectroscopy and theoretical studies", Physical Chemistry Chemical Physics, 20(44): 28319-28330, (2018).

[14] Amraoui, N. E., Messaoudi, A., Hammoutène, D., "Copper ion Cu (I) interaction effect on DNA nucleotides: DFT study”, Inorganic Chemistry Communications, 119: 108078, (2020).

[15] Amat, A., Rigau, J., Waynant, R.W., Ilev, I. K., Tomas, J., Anders, J. J., "Modification of the intrinsic fluorescence and the biochemical behavior of ATP after irradiation with visible and nearinfrared laser light", Journal of Photochemistry and Photobiology B Biology, 81(1): 26-32, (2005).

[16] Van Outersterp, R., "Structural characterization and activation of nature's fuels of life", Student Undergraduate Research E-journal, 2, (2016).

[17] Burke, R. M., Dessent, C. E., "Effect of cation complexation on the structure of a conformationally flexible multiply charged anion: stabilization of excess charge in the $\mathrm{Na}+$-Adenosine $5^{\prime}-$ Triphosphate dianion ion-pair complex", The Journal of Physical Chemistry A, 113(12): 26832692, (2009).

[18] Cercola, R., Matthews, E., Dessent, C. E. H., "Photoexcitation of Adenosine 5'-Triphosphate Anions in Vacuo: Probing the Influence of Charge State on the UV Photophysics of Adenine", The Journal of Physical Chemistry B, 121(22): 5553-5561, (2017).

[19] Wu, R., Hamlow, L., He, C., Nei, Y.w., Berden, G., Oomens, J., Rodgers, M., “The intrinsic basicity of the phosphate backbone exceeds that of uracil and thymine residues: protonation of the phosphate moiety is preferred over the nucleobase for pdThd and pUrd", Physical Chemistry Chemical Physics, 19(45): 30351-30361, (2017).

[20] Sabat, M., Cini, R., Haromy, T., Sundaralingam, M., "Crystal structure of the alpha, beta, gammatridentate manganese complex of adenosine 5'-triphosphate cocrystallized with 2,2'dipyridylamine", Biochemistry, 24(26): 7827-7833, (1985).

[21] Kennard, O., Isaacs, N.W., Coppola, J.C., Kirby, A. J., Warren, S., Motherwell, W. D., Watson, D. G., Wampler, D. L., Chenery, D. H., Larson, A. C., Kerr, K. A., Di Sanseverino, L. R., "Three dimensional structure of adenosine triphosphate", Nature, 225(5230): 333-336, (1970).

[22] Akola, J., Jones, R., "ATP hydrolysis in water- a density functional study", The Journal of Physical Chemistry B, 107(42): 11774-11783, (2003).

[23] Wang, P., Izatt, R. M., Oscarson, J.L., Gillespie, S.E., "H NMR study of protonation and mg (ii) coordination of AMP, ADP, and ATP at 25, 50, and $70^{\circ}$ C", The Journal of Physical Chemistry, 100(22): 9556-9560, (1996).

[24] Zhou, J., Lu, G., "Spectroscopy study on the noncovalent interactions in the binary and ternary systems of L-lysine, adenosine 5'-triphosphate and magnesium ions", Spectrochim Acta A Mol Biomol Spectrosc, 78(4): 1305-1309, (2011).

[25] Brøndsted Nielsen, S., Sølling, T.I., "Are conical intersections responsible for the ultrafast processes of adenine, protonated adenine, and the corresponding nucleosides?", ChemPhysChem, 6(7): 12761281, (2005).

[26] Wu, R.R., He, C.C., Hamlow, L.A., Nei, Y.W., Berden, G., Oomens, J., Rodgers, M.T., "N3 Protonation Induces Base Rotation of 2'-Deoxyadenosine-5'-monophosphate and Adenosine-5'monophosphate", The Journal of Physical Chemistry B, 120(20): 4616-4624, (2016). 
[27] Wu, R. R., He, C. C., Hamlow, L. A., Nei, Y. W., Berden, G., Oomens, J., Rodgers, M.T., "Protonation induces base rotation of purine nucleotides pdGuo and pGuo", Physical Chemistry Chemical Physics, 18(22): 15081-15090, (2016).

[28] Frisch, M. J., Trucks, G., Schlegel, H., Scuseria, G., Robb, M., Cheeseman, J., Scalmani, G., Barone, V., Mennucci, B., Petersson, G., "Gaussian 09, Revision D. 01”, Gaussian Inc.: Wallingford, CT, (2009).

[29] Shao, Y., Molnar, L., Jung, Y., Kussmann, J., Ochsenfeld, C., Brown, S., Gilbert, A., Slipchenko, L., Levchenko, S., O'Neill, D., "Spartan'08, Wavefunction, Inc. Irvine, CA”, Physical Chemistry Chemical Physics, 8: 3172-3191, (2006).

[30] Dereli, Ö., "Molecular structure and spectral (FT-IR, Raman) investigations of 3-aminocoumarin", Optics and Spectroscopy, 120(5): 690-700, (2016).

[31] Miertuš, S., Scrocco, E., Tomasi, J., "Electrostatic interaction of a solute with a continuum. A direct utilizaion of $\mathrm{AB}$ initio molecular potentials for the prevision of solvent effects", Chemical Physics, 55(1): 117-129, (1981).

[32] Tomasi, J., Mennucci, B., Cammi, R., "Quantum mechanical continuum solvation models", Chemical Reviews, 105(8): 2999-3093, (2005).

[33] Pakiari, A., Farrokhnia, M., Moradshahi, A., "Quantum chemical analysis of ATP, GTP and related compounds in gas phase", Journal of the Iranian Chemical Society, 7(1): 51-58, (2010).

[34] Kennard, O., Isaacs, N.W., Motherwell, W., Coppola, J., Wampler, D., Larson, A.t., Watson, D., "The crystal and molecular structure of adenosine triphosphate", Proceedings of the Royal Society of London. A. Mathematical Physical Sciences, 325(1562): 401-436, (1971).

[35] Sugawara, Y., Kamiya, N., Iwasaki, H., Ito, T., Satow, Y., "Humidity-controlled reversible structure transition of disodium adenosine 5'-triphosphate between dihydrate and trihydrate in a single crystal state", Journal of the American Chemical Society, 113(14): 5440-5445, (1991).

[36] Dittrich, M., Hayashi, S., Schulten, K., "On the mechanism of ATP hydrolysis in F1-ATPase", Biophysical Journal, 85(4): 2253-2266, (2003).

[37] Kurban, M., Gündüz, B., Göktaş, F., "Experimental and theoretical studies of the structural, electronic and optical properties of BCzVB organic material", Optik, 182: 611-617, (2019).

[38] Gündüz, B., Kurban, M., "Photonic, spectroscopic properties and electronic structure of PTCDI-C8 organic nanostructure", Vibrational Spectroscopy, 96: 46-51, (2018).

[39] Kurban, M., Sertbakan, T. R., Gündüz, B., “A combined experimental and DFT/TD-DFT studies on the electronic structure, structural and optical properties of quinoline derivatives", Journal of Molecular Modeling, 26: 1-7, (2020).

[40] Reed, J. L., "Electronegativity: chemical hardness I", The Journal of Physical Chemistry A, 101(40): 7396-7400, (1997).

[41] Parr, R.G., Pearson, R.G., "Absolute hardness: companion parameter to absolute electronegativity", Journal of the American Chemical Society, 105(26): 7512-7516, (1983).

[42] Pauling, L., The Nature of the Chemical Bond, Cornell University Press Ithaca, NY, (1960).

[43] Koopmans, T., "Über die Zuordnung von Wellenfunktionen und Eigenwerten zu den einzelnen Elektronen eines Atoms", Physica, 1(1-6): 104-113, (1934). 
[44] Roy, D. R., Parthasarathi, R., Padmanabhan, J., Sarkar, U., Subramanian, V., Chattaraj, P. K., "Careful scrutiny of the philicity concept", The Journal of Physical Chemistry A, 110(3): 10841093, (2006).

[45] Singh, N.B., Sarkar, U., "Structure, vibrational, and optical properties of platinum cluster: a density functional theory approach", Journal of Molecular Modeling, 20(12): 2537, (2014).

[46] Grimme, S., "Calculation of the electronic spectra of large molecules", Reviews in Computational Chemistry, 20: 153, (2004).

[47] Dreuw, A., Head-Gordon, M., "Single-reference ab initio methods for the calculation of excited states of large molecules", Chemical reviews, 105(11): 4009-4037, (2005).

[48] Varsano, D., Di Felice, R., Marques, M. A., Rubio, A., "A TDDFT study of the excited states of DNA bases and their assemblies", The Journal of Physical Chemistry B, 110(14): 7129-7138, (2006).

[49] Adamo, C., Jacquemin, D., "The calculations of excited-state properties with Time-Dependent Density Functional Theory", Chemical Society Reviews, 42(3): 845-856, (2013).

[50] Ito, A., Ito, T., "Absorption spectra of deoxyribose, ribosephosphate, atp and dna by direct transmission measurements in the vacuum-uv $(150-190 \mathrm{~nm})$ and far-uv $(190-260 \mathrm{~nm})$ regions using synchrotron radiation as a light source", Photochemistry and Photobiology, 44(3): 355-358, (1986).

[51] Hegyi, G., Kardos, J., Kovács, M., Málnási-Csizmadia, A., Nyitray, L., Pál, G., Radnai, L., Reményi, A., Venekei, I., "Introduction to practical biochemistry", ELTE Faculty of Natural Sciences, (2013).

[52] Carrasquilla, R., Bueno, O. N., "Time dependent density functional study of the absorption and emission spectra of 1,3-benzoxazole and three substituted benzoxazoles", Optica pura y aplicada, 45(3): 287-297, (2012).

[53] Cervantes-Navarro, F., Glossman-Mitnik, D., "DFT study of the effect of substituents on the absorption and emission spectra of Indigo", Chemistry Central Journal, 6(1): 70, (2012).

[54] Noguchi, T., Shiraki, T., Dawn, A., Tsuchiya, Y., Yamamoto, T., Shinkai, S., "Nonlinear fluorescence response driven by ATP-induced self-assembly of guanidinium-tethered tetraphenylethene", Chemical Communications, 48(65): 8090-8092, (2012). 